INPLASY

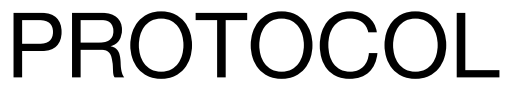

To cite: Zhong et al. Effectiveness and Safety of Acupuncture for Insomnia in COVID-19 Patients: A protocol of Systematic Review and Meta-analysis. Inplasy protocol 202230017. doi:

10.37766/inplasy2022.3.0017

Received: 03 March 2022

Published: 03 March 2022

Corresponding author:

Yanmei Zhong

1422311457@qq.com

Author Affiliation:

School of Medical Information

Engineering, Chengdu

University of Traditional

Chinese Medicine.

Support: This study is supported by Spe.

Review Stage at time of this submission: The review has not yet started.

Conflicts of interest:

None declared.

\section{Effectiveness and Safety of Acupuncture for Insomnia in COVID-19 Patients: A protocol of Systematic Review and Meta-analysis}

Zhong, Y1; Lu, H²; Liu, H³; Liu, L4; Wen, C5; Cheng, X6.

Review question / Objective: Our study aims to evaluate the efficacy and safety of acupuncture on insomnia in patients with COVID-19.

Eligibility criteria: (1)Type of participants: Patients diagnosed with COVID-19 together with insomnia. (Conforming to any recognized diagnostic criteria such as SARS-CoV-2 antigen detection tests and SARS-CoV-2 nucleic acid detection tests, regardless of patient's ethnicity, country, age, and course of the disease)(2)Type of interventions: The treatment group underwent acupuncture treatment, such as manual acupuncture, electroacupuncture, auricular acupuncture, scalp acupuncture and plum blossom needle, regardless of acupoint selection, treatment frequency, or course. The control group adopted conventional Western medicine treatment, placebo, sham, or no treatment.(3)Type of comparator: The control group adopted conventional treatment, medication, such as eszopiclone, zaleplon, zolpidem placebo, sham acupuncture, or no treatment.(4) Type of studies: Only randomized control trials (RCTs) studies will be included.

INPLASY registration number: This protocol was registered with the International Platform of Registered Systematic Review and Meta-Analysis Protocols (INPLASY) on 03 March 2022 and was last updated on 03 March 2022 (registration number INPLASY202230017).

\section{INTRODUCTION}

Review question / Objective: Our study aims to evaluate the efficacy and safety of acupuncture on insomnia in patients with COVID-19.

Condition being studied: Coronavirus disease 19 (COVID-19) refers to an infectious disease caused by the SARSCoV-2, whose primary clinical manifestations are elevated body temperature, cough, dyspnea, and malaise, and some critically ill patients may suffer from serious complications such as acute respiratory distress syndrome (ARDS) with an incubation period of 1-14 days. 
According to the latest data from the WHO, globally, as of 5:11 p.m. CET on March 2, 2022, there were $437,333,859$ confirmed cases of COVID-19, which includes $5,960,972$ deaths. Consequently, this unprecedentedly contagious pandemic has been a heavy health burden afflicting everyone. Previous studies have demonstrated that outbreaks of infectious diseases are associated with mental health symptoms and disorders (e.g., insomnia, depression, anxiety, post-traumatic stress disorder. Patients with COVID-19 during hospitalization often experienced significant insomnia triggered by loneliness during quarantine, anxiety and trepidation from death, which seriously hinders their physical recovery from COVID-19 and associated psychological problems. Acupuncture, one of the alternative therapies, has been extensively utilized in clinical practice to combat insomnia all over the world. A multitude of randomized controlled clinical trials has revealed the favorable effects of acupuncture in the treatment of insomnia. Several studies have demonstrated that acupuncture treatment outperformed conventional Western medication in terms of sleep disorder, not only in primary insomnia but also secondary insomnia, such as insomnia in patients with stroke, depression, anxiety. The prevalence of insomnia among COVID-19 patients triggers individuals to embrace non-pharmacological treatments. Although extensive research has been carried out on the benefits of acupuncture for insomnia, no single study exists that systematically evaluates the efficacy of acupuncture for insomnia in COVID-19 patients.

\section{METHODS}

Search strategy: The following databases will be searched to collate documents relating to acupuncture for COVID-19 combined with insomnia: Web of Science, PubMed, EMBASE, Ovid, The Cochrane Library, China Science and Technology Journal (VIP), Wanfang database, Chinese Biological Medical (CBM), and China National, Knowledge Infrastructure (CNKI). Gray literature will be obtained from
GreyNet, Clinical Trails, Chinese Cochrane Center and Chinese Clinical Trial Registry. Literature is limited to those published before March 1, 2022, whose study type is clinical randomized controlled trials (RCTs) only and there is no language limitation. The search terms involve COVID-19, insomnia, acupuncture, and clinical randomized controlled trials. The search strategy will be adjusted according to the characteristics of the databases. The management of all references will be performed by Endnote 20.0. Table 1 shows the search strategy of Pub-med.

Participant or population: Patients diagnosed with COVID-19 together with insomnia. (Conforming to any recognized diagnostic criteria such as SARS-CoV-2 antigen detection tests and SARS-CoV-2 nucleic acid detection tests, regardless of patient's ethnicity, country, age, and course of the disease).

Intervention: The treatment group underwent acupuncture treatment, such as manual acupuncture, electroacupuncture, auricular acupuncture, scalp acupuncture and plum blossom needle, regardless of acupoint selection, treatment frequency, or course. The control group adopted conventional Western medicine treatment, placebo, sham, or no treatment.

Comparator: (3)The control group adopted conventional treatment, medication, such as eszopiclone, zaleplon, zolpidem placebo, sham acupuncture, or no treatment.

Study designs to be included: Only randomized control trials (RCTs) studies will be included.

Eligibility criteria: (1)Type of participants: Patients diagnosed with COVID-19 together with insomnia. (Conforming to any recognized diagnostic criteria such as SARS-CoV-2 antigen detection tests and SARS-CoV-2 nucleic acid detection tests, regardless of patient's ethnicity, country, age, and course of the disease)(2)Type of interventions: The treatment group 
underwent acupuncture treatment, such as manual acupuncture, electroacupuncture, auricular acupuncture, scalp acupuncture and plum blossom needle, regardless of acupoint selection, treatment frequency, or course. The control group adopted conventional Western medicine treatment, placebo, sham, or no treatment.(3)Type of comparator: The control group adopted conventional treatment, medication, such as eszopiclone, zaleplon, zolpidem placebo, sham acupuncture, or no treatment. (4) Type of studies: Only randomized control trials (RCTs) studies will be included.

Information sources: Web of Science, PubMed, EMBASE, Ovid, the Cochrane Library, China Science and Technology Journal Database, Chinese Biomedical Literature Database(CBM) and China National Knowledge Infrastructure (CNKI), GreyNet, Clinical Trails, Chinese Cochrane Center and Chinese Clinical Trial Registry.

Main outcome(s): The effective rate will be our primary outcome.

Additional outcome(s): secondary outcomes are the Pittsburgh Sleep Quality Index score (PSQI), Insomnia Severity Index (ISI), Self-Rating Anxiety Scale (SAS), the total sleep time, the recurrence rate, and the adverse events.

Quality assessment / Risk of bias analysis: The risk of bias tool of the Cochrane Handbook 5.1.0. will be utilized by two authors independently to evaluate the quality of included trials, which involves the following indicators: the allocation sequence random, allocation concealment, blinding of participants and personnel, blinding of outcome assessment, incomplete outcome data, and other bias. And the risk of bias was rated as "low", "unclear" or "high".

Strategy of data synthesis: The statistical analysis will be achieved by RevMan 5.4.1. and STATA 15.0. The heterogeneity test will be carried out by 12 statics; 12 exceeding $50 \%$ indicates significant heterogeneity. The random-effects model will be applied when there is significant heterogeneity with 12 exceeding $50 \%$, while when 12 is less than $50 \%$, the fixed-effects model will be used. Risk ratio 13 with $95 \%$ confidence interval 12 will be used to determine dichotomous data, and weighted mean differences $(95 \% \mathrm{Cl})$ or standardized mean differences $(95 \% \mathrm{Cl})$ will be used to analyze the continuous data.

Subgroup analysis: When the heterogeneity test results are substantial (I2>50\%), RevMan 5.4.1. will perform subgroup analysis of age, gender, interventions, acupoints selection and treatment intervals.

Sensitivity analysis: Sensitivity analyses will be performed to verify the stability and reliability of the results of this study in accordance with the recommendations of the Cochrane Handbook. If the heterogeneity test of outcome is substantial $(12>50 \%)$, the sensitivity analysis will be conducted for identifying the source of homogeneity.

Country(ies) involved: China.

Keywords: COVID-19; insomnia; acupuncture; meta-analysis; protocol.

Contributions of each author:

Author 1 - Yanmei Zhong - Author 1 drafted the manuscript and was responsible for conceptualization, methodology and project administration.

Email: 1422311457@qq.com

Author 2 - Haizhen Lu - The author was in charge of data curation.

Email: luhaizhen@stu.cdutcm.edu.cn

Author 3 - Han Liu - Author 3 was responsible for collecting study data.

Email: 906499322@qq.com

Author 4 - Lu Liu - Author 4 was responsible for the methodology of the study.

Email: 756132259@qq.com

Author 5 - chuanbiao Wen - Author 5 was responsible for funding acquisition.

Email: 228237222@qq.com

Author 6 - Xiaoen Cheng - Author 6 was responsible for funding acquisition.

Email: 1157778219@qq.com 